\title{
Biased Diffusion in Confined Media
}

\author{
P. S. Burada*, G. Schmid*, D. Reguera ${ }^{\dagger}$, J. M. Rubí ind $^{*}$ and Hänggi* \\ *Institut für Physik, Universität Augsburg, Universitätsstr. 1, D-86135 Augsburg, Germany \\ ${ }^{\dagger}$ Departament de Física Fonamental, Facultat de Física, Universidad de Barcelona, Diagonal 647, \\ E-08028 Barcelona, Spain
}

\begin{abstract}
We study biased, diffusive transport of Brownian particles through narrow, spatially periodic structures in which the motion is constrained in lateral directions. The problem is analyzed using the Fick-Jacobs equation in which the effect of the lateral confinement is replaced by an entropic barrier. The validity of this approximation, being based on the assumption of an instantaneous equilibration of the particle distribution in the cross-section of the structure, is analyzed by comparing the different time scales that characterize the problem. A validity criterion is established in terms of the shape of the structure and of the applied force. It is analytically corroborated and verified by numerical simulations that the critical value of the force up to which this description holds true scales as the square of the periodicity of the structure. The criterion can be visualized by means of a diagram representing the regions where the Fick-Jacobs description becomes inaccurate in terms of the scaled force versus the periodicity of the structure.
\end{abstract}

Keywords: Entropic transport; diffusion; Brownian motion; confined systems; nanoporous materials; Fick-Jacobs equation

PACS: $05.60 . \mathrm{Cd}, 05.40 . \mathrm{Jc}, 02.50 . \mathrm{Ey}$

\section{INTRODUCTION}

The diffusion of Brownian objects in confined media plays a fundamental role in many transport phenomena such as those taking place in biological cells, ion channels, nanoporous materials and microfluidic devices. The uneven shape of these structures regulates the transport of particles yielding important effects exhibiting peculiar properties. The results have implications in processes such as catalysis, osmosis and particle separation $[1,2,3,4,5,6,7,8]$.

The motion of the particles through these quasi-one-dimensional structures can in principle be analyzed by means of the standard protocol of solving the Smoluchowski equation with the appropriate boundary conditions imposed. Whereas this method has been very successful when the boundaries of the system possess a regular shape, the challenge to solve the boundary value problem in the case of uneven boundaries represents typically a very difficult task. A way to circumvent this difficulty consists in coarsening the description by reducing the dimensionality of the system, keeping only the main direction of transport, but taking into account the irregular nature of these boundaries by means of an entropic potential. The resulting kinetic equation for the probability distribution, the Fick-Jacobs (F-J) equation, is similar in form to the Smoluchowski equation, but now contains an entropic term. The entropic nature of this term leads to a genuine dynamics which is very different from that observed when the potential has an energetic origin [9]. It has been shown that the F-J equation can provide a very accurate description of entropic transport in 2D and 3D channels of varying cross-section $[9,10]$. 


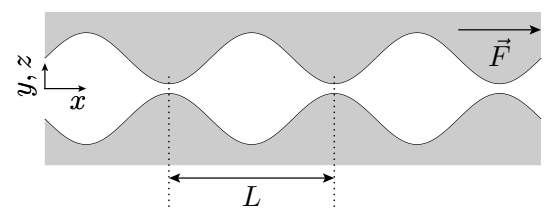

FIGURE 1. Schematic diagram of the channel confining the motion of the biased Brownian particles. The shape of the channel is described by $\omega(x)=\sin (2 \pi x / L)+1.02$.

However, the derivation of the F-J equation entails a tacit approximation: The particle distribution in the transverse direction is assumed to equilibrate much faster than in the main (unconstrained) direction of transport. This equilibration justifies the coarsening of the description leading in turn to a simplification of the dynamics, but raises the question about its validity when an external force is applied.

Our objective with this work is to investigate in greater detail the F-J approximation for biased diffusion and to set up a corresponding criterion describing its regime of validity. We will analyze the biased movement of Brownian particles in 2D periodic channels of varying cross section and formulate different criteria for the validity of such a F-J description.

\section{DIFFUSION IN CONFINED SYSTEMS AND THE FICK-JACOBS EQUATION}

The dynamics of a Brownian particle through a pore or a channel (like the one depicted in Fig. 1) in the overdamped limit and in the presence of an external potential $V(\vec{r})$ is governed by the equation:

$$
\eta \frac{\mathrm{d} \vec{r}}{\mathrm{~d} t}=-\vec{\nabla} V(\vec{r}(t))+\sqrt{\eta k_{\mathrm{B}} T} \vec{\xi}(t) .
$$

where $\vec{r}$ is the two or three dimensional position vector of the particle, $\eta$ is its friction coefficient, $k_{\mathrm{B}}$ the Boltzmann constant, $T$ the temperature, and $\vec{\xi}(t)$ is a Gaussian white noise with zero mean and correlation $\left\langle\xi_{i}(t) \xi_{j}\left(t^{\prime}\right)\right\rangle=2 \delta_{i j} \delta\left(t-t^{\prime}\right)$ for $i, j=x, y, z$.

In addition to Eq. (1), the full problem is set up by imposing reflecting boundary conditions at the channel walls. In this work we focus on the case of a symmetric 2D channel where the force is constant and directed along the axis, cf. Fig. 1. The halfwidth of the $2 \mathrm{D}$ channel is given by a periodic function $\omega(x)$, i.e. $\omega(x+L)=\omega(x)$ for all $x$. For an arbitrary form of $\omega(x)$, the boundary value problem associated to the Langevin equation above (or the corresponding Fokker-Planck equation) is very difficult to solve. Despite the inherent complexity of this problem an approximate solution can be found using the Fick-Jacobs approximation, which results in an effective onedimensional description where geometric constraints and bottlenecks are considered as entropic barriers $[9,13,14,10,15]$.

In the presence of a constant force $F$ along the direction of the channel, it can be shown $[10,9]$ that the dynamics of a confined Brownian particle can be described by the 
F-J equation

$$
\frac{\partial P}{\partial t}=\frac{\partial}{\partial x}\left(D(x) \frac{\partial P}{\partial x}+\frac{D(x)}{k_{\mathrm{B}} T} \frac{\partial A(x)}{\partial x} P\right)
$$

where $P(x, t)$ is the probability distribution function along the axis of the 2D channel, $A(x):=E-T S=-F x-T k_{\mathrm{B}} \ln h(x)$ is the free energy, with $E=V=-F x$ denoting the energy contribution and $S=k_{\mathrm{B}} \ln h(x)$ the entropy contribution, and $h(x):=2 \omega(x) / L$ is the dimensionless width. This equation can be obtained from the 2D Smoluchowski equation after elimination of $y$ a coordinate, and it is valid for $\left|\omega^{\prime}(x)\right| \ll 1$. It has been shown that the introduction of an effective $x$-dependent diffusion coefficient

$$
D(x)=\frac{D_{0}}{\left(1+\omega^{\prime}(x)^{2}\right)^{\alpha}},
$$

where $\alpha=1 / 3,1 / 2$ for two and three dimensions, respectively, can considerably improve the accuracy of this description, thus extending its validity to more winding structures $[10,15]$. In the presence of very strong applied bias, and for more winding structures, however, the F-J equation becomes inaccurate. In the present work we present further numerical and analytical results and will set up tailored criteria under which the F-J approximation assumes good validity.

In particular, we will focus on how accurate the F-J equations describes the transport through the nonlinear mobility, which is defined as the ratio of the average particle current $\langle\dot{x}\rangle$ and the applied force $F$. The nonlinear mobility for a $2 \mathrm{D}$ channel with a shape defined by $\omega(x)$ depends on a single, dimensionless scaling parameter [9]:

$$
f:=\beta F L
$$

and it is given by

$$
\mu(f) \eta=\frac{1}{f} \frac{1-\exp (-f)}{\int_{0}^{1} \mathrm{~d} z I(z, f)}
$$

where

$$
I(z, f)=\exp (f z) h(L z) \int_{z}^{z+1} \mathrm{~d} z^{\prime} \exp \left(-f z^{\prime}\right) h^{-1}\left(L z^{\prime}\right)\left[1+\left(\frac{1}{L} \frac{\mathrm{d} \omega\left(L z^{\prime}\right)}{\mathrm{d} z^{\prime}}\right)^{2}\right]^{\alpha}
$$

\section{VALIDITY OF THE FICK-JACOBS DESCRIPTION IN PRESENCE OF A CONSTANT BIAS}

The reduction of dimensionality done implicitly in the formulation of the F-J equation relies on the assumption of equilibration in the transverse direction. An estimate of the conditions under which equilibration occurs can be made by analyzing the different time scales involved in the problem. One is diffusion in the transverse direction over a distance $\Delta y$, whose time scale is $\tau_{y}=\frac{\Delta y^{2}}{2 D_{0}}$. Similarly, the time scale associated to diffusion in the axial direction is $\tau_{x}=\frac{\Delta x^{2}}{2 D_{0}}$. The third time scale is related to the drift (ballistic motion) associated to the force, i.e. $\tau_{d r i f t}=\frac{L \Delta x}{D_{0} f}$. 
In the absence of an external force, equilibration in the transverse direction occurs if $\tau_{y} / \tau_{x} \ll 1$. This results in the condition:

$$
\frac{\Delta y^{2}}{\Delta x^{2}} \sim \omega^{\prime}(x)^{2} \ll 1,
$$

which constitutes the validity criterion of the F-J approach such as put forward by Zwanzig [14].

In presence of a force along the axis, equilibration in the transverse direction demands that the condition $\frac{\tau_{y}}{\tau_{d r i f t}} \ll 1$ also holds. Consequently,

$$
\frac{f \Delta y^{2}}{2 L \Delta x} \sim \frac{2 f \omega(x)^{2}}{L^{2}} \ll 1,
$$

where in the second step we have replaced the characteristic distances $\Delta y$ by the width $2 \omega(x)$, and $\Delta x$ by $L$.

A general estimate of the criteria that has to be satisfied can be put forward by considering the sum of the two ratios, cf. Eqs. (7) and (8), i.e.:

$$
\omega^{\prime}(x)^{2}+\frac{2 f \omega(x)^{2}}{L^{2}} \ll 1
$$

We can now get a global criteria by averaging the previous local criterion over the period $L$ of the channel and by defining a critical force value $f_{c}$, for which the inequality becomes an equality. This critical force value then reads:

$$
f_{c}=\frac{L^{2}}{2\left\langle\omega(x)^{2}\right\rangle}\left(1-\left\langle\omega^{\prime}(x)^{2}\right\rangle\right) .
$$

Eq. (10) provides an estimate of the minimum forcing beyond which the F-J description is expected to fail in providing an accurate description of the dynamics of system, and indicates that the critical force scales asymptotically as $L^{2}$ (for a fixed overall shape of the channel).

\section{NUMERICAL SIMULATIONS FOR A 2D CHANNEL}

In order to check the consistency of the criteria proposed and the validity of the F$\mathrm{J}$ description in the presence of a force, we have compared the analytical result for the scaled nonlinear mobility obtained in Eq. (5) with the simulation results of the overdamped Langevin dynamics in Eq. (1) for the 2D periodic channel sketched in Fig. 1, for different values of the periodicity $L$.

In Fig. 2 we illustrate the regions where the F-J approximation is accurate when compared with the simulations. The lines in this figure depict the critical values of the scaled force, $f_{c}$, beyond which the relative error in the mobility exceeds a certain value, as a function of the periodicity $L$. We have verified that he critical value of the force depends quadratically on the periodicity $L^{2}$, as predicted. Thus, it is possible to 


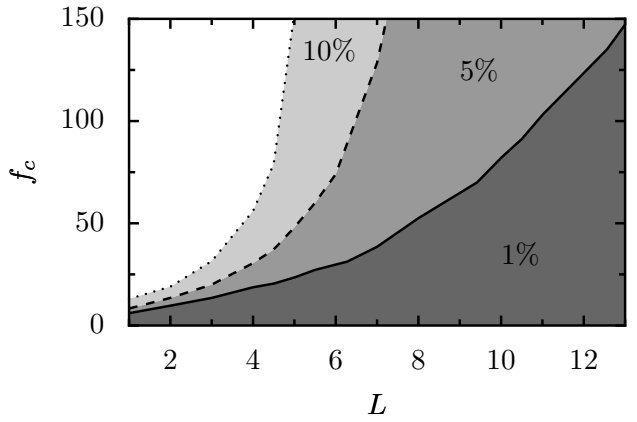

FIGURE 2. The validity diagram of the Fick-Jacobs approximation for biased diffusion is obtained upon a comparison between the precise numerics with the approximative analytic solution, cf. Eq. (5) for a $2 \mathrm{D}$ channel with boundary function $\omega(x)=\sin (2 \pi x / L)+1.02$. The dependence of the critical value of the scaling parameter on the periodicity is depicted for three different relative errors; $1 \%$ : solid line, 5\%: dashed line and 10\%: dotted line. Below these limiting lines the analytic treatment agrees within the corresponding prescribed relative error.

provide an accurate result by using the analytic solution over a wide range of the scaled parameter and the periodicity.

From our simulations, we can actually analyze the validity of the hypotheses of equilibration in the transverse direction on which the F-J description relies. In Fig. 3, we show the steady state distribution of a certain number of Brownian particles for different values of the scaled force $f$. As the force increases, we can clearly see how the particles are not homogeneously distributed in $y$-direction, evidencing the failure of the equilibration assumption. This effect is specially dramatic in Fig. 3(lower right panel), where the force is so strong that the particles do not fully explore the available space in $y$-direction. In this situation, the influence of the entropic barriers practically disappears and a deterministic treatment of the problem leads to adequate results.

\section{CONCLUSIONS}

In this work we have investigated the validity conditions under which the Fick-Jacobs approximation provides an accurate description of the biased diffusion of Brownian particles in 2D and 3D confined systems. We have established a validity criterion formulated in terms of the sinuosity of the channel $\omega(x)$, as done in the unbiased case $[13,14,10,15]$, and on the scaling parameter that causes forced diffusion. This scaling parameter compares the work done on a particle travelling a distance equal to the spatial period $L$ of the channel with the available thermal energy. Interestingly, the critical value of this scaling parameter up to which Fick-Jacobs equation holds depends on the square of the period. This dependence follows from the analysis of the different time scales that rule the biased, diffusive dynamics. We have constructed a validity diagram showing the region of parameters (spanned by $f$ and $L$ ) in which the Fick-Jacobs approximation describes the overdamped diffusive transport accurately cf. Fig. 2. We have also investigated numerically the conditions for a fast equilibration in the transverse direction which 

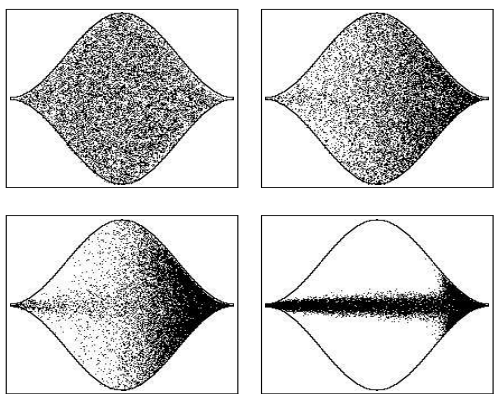

FIGURE 3. Steady-state particle distribution mapped into a single period of the $2 \mathrm{D}$ channel defined by the boundary function $\omega(x)=\sin (2 \pi x / L)+1.02$ with $L=1$ for four different values of the dimensionless scaling parameter $f$ : upper left panel: $f=0.2$; upper right panel: $f=3.0$; lower left panel: $f=7.0$; lower right panel: $f=50$.

is vital for the accurateness of the Fick-Jacobs approximation. The results presented evidence the usefulness of the Fick-Jacobs description with a spatially-dependent diffusion coefficient at small applied bias.

\section{ACKNOWLEDGMENTS}

This work has been supported by the DGiCYT under Grant No BFM2002-01267 (D.R.), ESF STOCHDYN project (G.S., D.R., J.M.R., P.H.), the Alexander von Humboldt Foundation (J.M.R.), the Volkswagen Foundation (project I/80424, P.H.), the DFG via research center, SFB-486, project A10 (G.S., P.H.) and via the project no. 1517/26-1 (P.S.B., P.H.), and by the Nanosystems Initiative Munich (P.H.).

\section{REFERENCES}

1. B. Hille, Ion Channels of Excitable Membranes (Sinauer, Sunderland, 2001).

2. R.M. Barrer, Zeolites an Clay Minerals as Sorbents and Molecular Sieves (Academic Press, London, 1978).

3. T. Chou and D. Lohse, Phys. Rev. Lett. 82, 3552 (1999).

4. L. Liu, P. Li, and S.A. Asher, Nature 397, 141 (1999).

5. Z. Siwy, I.D. Kosinska, A. Fulinski, C.R. Martin,Phys. Rev. Lett. 94, 048102 (2005).

6. A.M. Berezhkovskii and S.M. Bezrukov, Biophys. J. 88, L17(2005).

7. C. Kettner, P. Reimann, P. Hänggi, and F. Müller, Phys. Rev. E 61, 312 (2000).

8. S. Matthias and F. Müller, Nature 424, 53 (2003).

9. D. Reguera, G. Schmid, P.S. Burada, J.M. Rubí, P. Reimann, and P. Hänggi, Phys. Rev. Lett. 96, 130603 (2006).

10. D. Reguera and J.M. Rubí, Phys. Rev. E 64, 061106 (2001).

11. H. Risken, The Fokker-Planck equation, 2nd ed. (Springer, Berlin, 1989).

12. P. Hänggi and H. Thomas,Phys. Rep. 88, 207 (1982).

13. M.H. Jacobs, Diffusion Processes (Springer, New York, 1967).

14. R. Zwanzig, J. Phys. Chem. 96, 3926 (1992).

15. P. Kalinay and J.K. Percus, Phys. Rev. E 74, 041203 (2006). 